gr-qc/0409003

\title{
Decay Rate and Low-Energy Near-Horizon Dynamics of Acoustic Black Holes
}

\author{
Sung-Won Kim ${ }^{a *}$, Won Tae $\mathrm{Kim}^{b \dagger}$, and John J. $\mathrm{Oh}^{a, c \ddagger}$ \\ ${ }^{a}$ Department of Science Education and Basic Science Research Institute, \\ Ewha Women's University, Seoul 120-750, Korea \\ ${ }^{b}$ Department of Physics and Basic Science Research Institute, \\ Sogang University, C.P.O. Box 1142, Seoul, Korea \\ ${ }^{c}$ Department of Physics, University of Waterloo, Waterloo, ON, N2L 3G1, Canada
}

\begin{abstract}
We study the low-energy dynamics of an acoustic black hole near the sonic horizon. For the experimental test of black hole evaporation in the laboratory, the decay rate (greybody factor) of the acoustic black hole (sonic hole) can be calculated by the usual low-energy perturbation method. As a consequence, we obtain the decay rate of the sonic horizon from the absorption and the reflection coefficients. Moreover, we show that the thermal emission from the sonic horizon is only proportional to a control parameter which describes the velocity of the fluid.
\end{abstract}

PACS : 04.62.+v, 04.80.Cc, 47.90.+a

Typeset using REVTEX

\footnotetext{
*email:sungwon@mm.ewha.ac.kr

†email:wtkim@mail.sogang.ac.kr

‡email:j4oh@sciborg.uwaterloo.ca
} 
One of the most attractive aspects of general relativity is that it predicts the existence of black holes. Classically, they can absorb all sort of matter through the extremely strong gravitational field, and even light cannot escape from them. For this reason, they are black to all observers outside them. Their quantum mechanical properties are quite different from their classical ones. The surprising prediction that quantum black holes are no longer black was announced in 1974 [1], thereafter the quantum mechanical properties of them remain fascinating but problematic to theoretical and experimental physicists. From the viewpoint of quantum field theory in a curved spacetime background, they can emit thermal radiation proportional to the surface gravity at their horizons. This theoretical result, called "Hawking radiation", used to be asked if it is possible to verify it from experimental study within the framework of astrophysical phenomenology.

In 1981 Unruh proposed a possibility of an experimental test for the black hole evaporation [2], which opened a new branch of black hole physics [3-12]. He considered an irrotational inviscid fluid and sound wave propagating in the medium. According to Ref. [2], the linearized small perturbation of wave equations in the fluid leads to an equation of motion for a massless scalar field in the geometrical background that is similar to the black hole metric. There exists a trapped region that the sound wave cannot escape as the fluid velocity exceeds the sound velocity (hypersonic flow), which is called a "sonic horizon" when the fluid velocity is equals to that of the sound wave. This hypersonic flow system reveals more aspects of the acoustic black hole with the help of the analogy of black hole thermodynamics. By quantizing the sound field (phonons), the sonic hole (dumb hole) can emit sound waves with the thermal temperature given by

$$
T=\frac{\hbar}{2 \pi}\left[\frac{\partial v^{r}}{\partial r}\right]_{\text {horizon }},
$$

where $\hbar$ is a Planck constant and $v^{r}$ is a fluid velocity along the radial direction.

In this paper, we shall investigate the decay rate of sonic black holes from the nearhorizon low-energy dynamics, which is expected to give an experimental suggestion of detecting the thermal radiation. The low-energy perturbation analysis of phonons leads to an 
exact derivation of the absorption cross section and decay rate of the sonic horizon. As a consequence, those results depend only upon a control parameter that describes the velocity of the given fluid associated with the surface gravity on the horizon, which agrees with Unruh's prediction of thermal radiation.

Let us briefly review the hypersonic flow and acoustic black holes following Ref. [2]. The irrotational inviscid fluid flow is described by the following three equations,

$$
\begin{aligned}
& \vec{\nabla} \times \vec{v}=0, \\
& \rho\left[\frac{\partial}{\partial t} \vec{v}+(\vec{v} \cdot \vec{\nabla}) \vec{v}\right]=-\vec{\nabla} p-\rho \vec{\nabla} \Phi, \\
& \frac{\partial}{\partial t} \rho+\vec{\nabla} \cdot(\rho \vec{v})=0,
\end{aligned}
$$

where $\vec{v}$ is a velocity of the flow, $p$ is a pressure that is a function of the density $\rho$, and $\Phi$ is an external potential. Note that the first equation describes an irrotational flow, the second and the third ones are Eulerian and continuity equations, respectively. These can be linearized in the vicinity of some mean flow solution with $\xi=\xi_{0}+\bar{\xi}$ and $\psi=\psi_{0}+\bar{\psi}$ redefining the fields as $\vec{\nabla} G(\xi)=\vec{\nabla} p / \rho, \rho=e^{\xi}$, and $\vec{v}=\vec{\nabla} \psi$, where the barred parameters stand for the small fluctuations around the vacuum solutions with a zero label. Combining these linearized equations gives the massless scalar field equation describing the sound wave in the curved spacetime background

$$
\frac{1}{\sqrt{-g}} \partial_{\mu}\left(\sqrt{-g} g^{\mu \nu} \partial_{\nu} \bar{\psi}\right)=0
$$

with the background metric,

$$
g_{\mu \nu}=\frac{\rho_{0}}{c}\left(\begin{array}{cc}
-c^{2}+v_{0}^{2} & -v_{0}^{i} \\
-v_{0}^{j} & \delta_{i j}
\end{array}\right),
$$

where $i, j=1,2,3$ and $c$ is a local velocity of sound defined as $c^{2}=G^{\prime}(\xi)$. Following Ref. [2], we assume $c$ to be constant for convenience. ${ }^{1}$. Provided that the metric, (4),

\footnotetext{
${ }^{1}$ This is assumed to be constant since the role of sound in the fluid is similar to that of light in gravity, aside from the easy treatment of the remaining calculations.
} 
is spherically symmetric and stationary, we can change the time coordinate $t$ into $\tau=$ $t+\int d r\left(v_{0}^{r}(r) /\left(c^{2}-v_{0}^{r 2}(r)\right)\right.$, which yields a diagonalized metric,

$$
(d s)^{2}=\frac{\rho_{0}}{c}\left[-\left(c^{2}-v_{0}^{r 2}\right) d \tau^{2}+\frac{c^{2} d r^{2}}{c^{2}-v_{0}^{r 2}}+r^{2} d^{2} \Omega\right] .
$$

The coordinate transformation does not affect the remaining physical result although the metric is a coordinate-dependent quantity. We, therefore, can make it deform for convenience. Of course, if the metric had been transformed in the scalar field equation of Eq. (3), then the same diagonalized metric would have been obtained. The metric (5) has a sonic horizon at $c^{2}=v_{0}^{r 2}$, which coincides with the sonic fluid flow while the hypersonic flow $\left(c^{2}<v_{0}^{r 2}\right)$ prevents the sound wave from propagating and the sound wave eventually does not escape from the horizon.

If the flow smoothly exceeds the velocity of sound at the sonic horizon $r=r_{s h}$, the velocity can be expanded as $v_{0}^{r}(r)=-c+a\left(r-r_{s h}\right)+\mathcal{O}\left(r-r_{s h}\right)^{2}$, which yields (up to the first order of $r$ )

$$
c^{2}-v_{0}^{r 2} \approx 2 a c\left(r-r_{s h}\right),
$$

where $a$ is a control parameter associated with the velocity of fluid defined as $\left.(\vec{\nabla} \cdot \vec{v})\right|_{r=r_{s h}}$. Note that the parameter $a$ is related to the surface gravity up to some proportional factor, which will be shown later. The resulting metric, therefore, becomes

$$
(d s)^{2}=\frac{\rho_{0}}{c}\left[-2 a c\left(r-r_{s h}\right) d \tau^{2}+\frac{c d r^{2}}{2 a\left(r-r_{s h}\right)}+r^{2} d^{2} \Omega\right],
$$

which is similar to the near-horizon behavior of the Schwarzschild black hole as discussed in Ref. [2].

Our starting point is the field equation of the sound wave, Eq. (3), under the nearhorizon metric background of Eq. (7). Using the separation of variables, $\bar{\psi}(\tau, r, \theta, \varphi)=$ $u(r) \Theta(\theta) e^{i(m \varphi-\omega \tau)}$, the radial equation of Eq. (3) is written as

$$
\left(r-r_{s h}\right) \partial_{r}^{2} u(r)+\left[1+\frac{2}{r}\left(r-r_{s h}\right)\right] \partial_{r} u(r)-\left(\frac{c \ell(\ell+1)}{2 a r^{2}}-\frac{\omega^{2}}{4 a^{2}\left(r-r_{s h}\right)}\right) u(r)=0 .
$$


What we wish to do is to approximate the wave equation in the Schwarzschild geometry by another unphysical wave equation that has the same behavior near the sonic horizon. Even though the unphysical wave equation has the tremendous advantage that it is analytically solvable, we will work within the first order of the approximation, because the near-horizon limit of the Schwarzschild metric gives the simplified Eqs. (7) and (8).

From the change of variables, $0 \leq z=1-r_{s h} / r \leq 1$, the radial equation (8) becomes

$$
z(1-z) \partial_{z}^{2} u(z)+\partial_{z} u(z)-\left[A-\frac{B}{z(1-z)}\right] u(z)=0,
$$

where $A=c \ell(\ell+1) / 2 a r_{s h}$ and $B=\omega^{2} / 4 a^{2}$. Now let us define $u(z) \equiv z^{\alpha}(1-z)^{\beta} h(z)$ in order to remove singularities at $z=0$ and $z=1$, which leads to

$$
z(1-z) \partial_{z}^{2} h(z)+[1+2 \alpha-2(\alpha+\beta) z] \partial_{z} h(z)-[A+(\alpha+\beta)(\alpha+\beta-1)] h(z)=0,
$$

and the parameters $\alpha$ and $\beta$ are determined by $\alpha= \pm i \sqrt{B}$ and $\beta=1 \pm \sqrt{1-B}$, respectively. The solution of Eq. (10) is simply given by the hypergeometric function $F$ as

$$
\begin{gathered}
u(z)=C_{1} z^{\alpha}(1-z)^{\beta} F\left(-\frac{1}{2}+\alpha+\beta-\frac{1}{2} \sqrt{1-4 A},-\frac{1}{2}+\alpha+\beta+\frac{1}{2} \sqrt{1-4 A} ; 1+2 \alpha ; z\right) \\
+C_{2} z^{-\alpha}(1-z)^{\beta} F\left(-\frac{1}{2}-\alpha+\beta-\frac{1}{2} \sqrt{1-4 A},-\frac{1}{2}-\alpha+\beta+\frac{1}{2} \sqrt{1-4 A} ; 1-2 \alpha ; z\right) .
\end{gathered}
$$

Note that since this solution is symmetric for changing $\alpha$ into $-\alpha$, we take the plus sign of $\alpha$ for convenience. In $z \rightarrow 0$ limit, Eq. (11) becomes

$$
u_{\text {near }}(r) \approx C_{\text {out }} \exp \left[i \frac{\omega}{2 a} \ln \left(1-\frac{r_{s h}}{r}\right)\right]+C_{\text {in }} \exp \left[-i \frac{\omega}{2 a} \ln \left(1-\frac{r_{s h}}{r}\right)\right],
$$

where $C_{\text {out }} \equiv C_{1}$ and $C_{\text {in }} \equiv C_{2}$. At this stage, the boundary condition can be imposed with an appropriate physical situation, and here we take $C_{\text {out }}=0$. The configuration of the boundary condition that we are considering is shown in the FIG. 1. Using the $z \rightarrow 1-z$ transformation of hypergeometric function [13],

$$
\begin{aligned}
F(l, m ; n ; z) & =\frac{\Gamma(n) \Gamma(n-l-m)}{\Gamma(n-l) \Gamma(n-m)} F(l, m ; l+m-n+1 ; 1-z) \\
& +(1-z)^{n-l-m} \frac{\Gamma(n) \Gamma(l+m-n)}{\Gamma(l) \Gamma(m)} F(n-l ; n-m ; n-l-m+1 ; 1-z),
\end{aligned}
$$




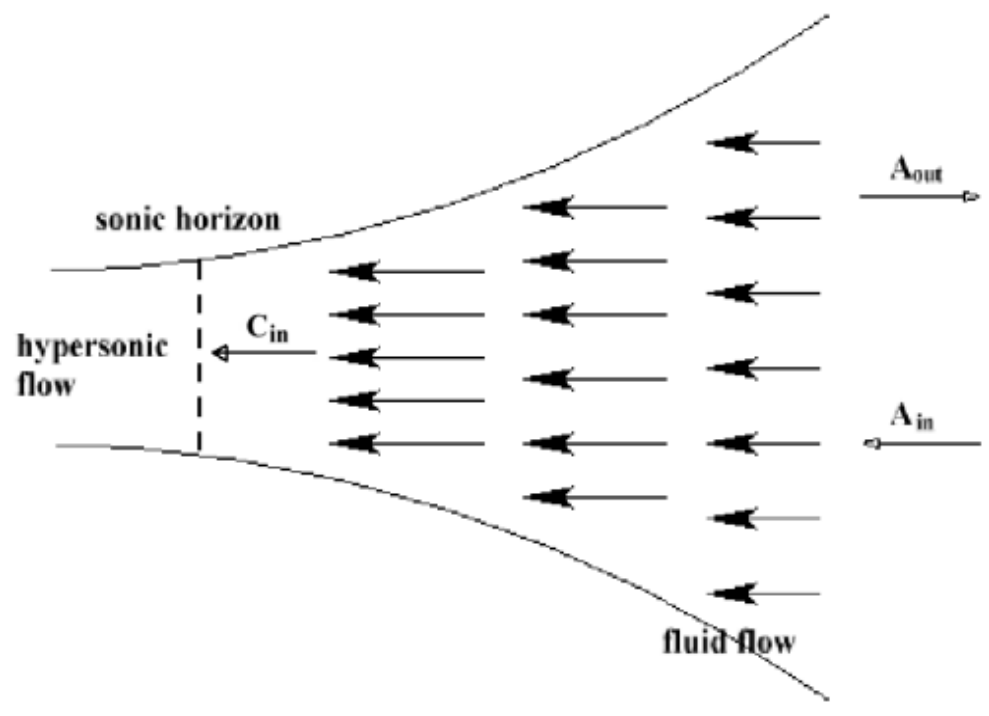

FIG. 1. Configuration of the acoustic black holes and the boundary condition

the far-region solution of Eq. (11) for $z \rightarrow 1$ is given by

$$
\begin{aligned}
u_{\text {far }}(r) & \approx C_{\text {in }}\left[\frac{\Gamma(1-2 \alpha) \Gamma(2-2 \beta) r_{s h}^{\beta}}{\Gamma\left(\frac{3}{2}-\alpha-\beta+\frac{1}{2} \sqrt{1-4 A}\right) \Gamma\left(\frac{3}{2}-\alpha-\beta-\frac{1}{2} \sqrt{1-4 A}\right)} r^{-\beta}\right. \\
& \left.+\frac{\Gamma(1-2 \alpha) \Gamma(2 \beta-2) r_{s h}^{2-\beta}}{\Gamma\left(-\frac{1}{2}-\alpha+\beta-\frac{1}{2} \sqrt{1-4 A}\right) \Gamma\left(-\frac{1}{2}-\alpha+\beta+\frac{1}{2} \sqrt{1-4 A}\right)} r^{\beta-2}\right] .
\end{aligned}
$$

This far region solution is symmetric for changing $\beta$ into $2-\beta$, which means that we can take the plus sign of $\beta$ for convenience. Since $\beta=1+\sqrt{4 a^{2}-\omega^{2}} / 2 a$, we consider the real value case of $\beta$, which signifies that the control parameter of the fluid velocity, $a$, is much greater than the energy of sound wave, $4 a^{2} \geq \omega^{2}$. This is nothing but a low-energy situation that we are considering.

On the other hand, the asymptotic behavior of Eq. (8) produces the wave equation at boundary,

$$
r^{2} \partial_{r}^{2} u_{\mathrm{B}}(r)+3 r \partial_{r} u_{\mathrm{B}}(r)+\frac{\omega^{2}}{4 a^{2}} u_{\mathrm{B}}(r)=0,
$$

which can be solved by

$$
u_{\mathrm{B}}(r)=A_{1} r^{-\beta}+A_{2} r^{\beta-2} .
$$


The coefficients, $C_{\text {in }}$ and $A_{1} / A_{(2)}$, can be exactly matched by comparing Eq. (14) with Eq. (16). Note that $\beta=2$ at low frequency region since $4 a^{2}>>\omega^{2}$. The "ingoing" and "outgoing" waves (denoted by $A_{\text {in }}$ and $A_{\text {out }}$, respectively) of Eq. (16) can be decomposed by introducing the redefinition of amplitudes,

$$
\begin{aligned}
& A_{1}=i L\left(A_{\text {in }}-A_{\text {out }}\right), \\
& A_{2}=A_{\text {in }}+A_{\text {out }},
\end{aligned}
$$

which leads to

$$
u_{\mathrm{B}}(r)=u_{\mathrm{B}}^{\mathrm{in}}(r)+u_{\mathrm{B}}^{\mathrm{out}}(r)=A_{\mathrm{in}}\left(1+i \frac{L}{r^{2}}\right)+A_{\mathrm{out}}\left(1-i \frac{L}{r^{2}}\right)
$$

where $L$ is a positive dimensionless constant, which will be taken to be independent of the energy $\omega[14,16]$. Note that $L$ can be chosen so that the absorption cross-section has the value of the area of the black hole as $\omega \rightarrow 0[14,17]$ or it has the usual value of the Hawking temperature [16]. However, in this approach, we shall take into account a different requirement of determining a positive dimensionless constant $L$. The fact that $\mathcal{A}+\mathcal{R}=1$ can determine the dimensionless constant $L$ in our configuration shown in FIG. 1.

The absorption $(\mathcal{A})$ and the reflection $(\mathcal{R})$ coefficients can be defined by the ratio of "ingoing" and "outgoing" fluxes,

$$
\mathcal{A}=\left|\frac{\mathcal{F}_{\text {near }}^{\text {in }}}{\mathcal{F}_{\mathrm{B}}^{\text {in }}}\right|, \quad \mathcal{R}=\left|\frac{\mathcal{F}_{\mathrm{B}}^{\text {out }}}{\mathcal{F}_{\mathrm{B}}^{\text {in }}}\right| .
$$

respectively. It is easy to derive the flux from Eq. (8) and it becomes

$$
\mathcal{F}=\frac{2 \pi}{i} r^{2} g^{r r}\left(u^{*}(r) \partial_{r} u(r)-u(r) \partial_{r} u^{*}(r)\right)
$$

The reflection coefficient can be expressed in terms of the matched coefficients in Eqs. (14) and (16) as

$$
\mathcal{R}=\left|\frac{\mathcal{F}_{\mathrm{B}}^{\text {out }}}{\mathcal{F}_{\mathrm{B}}^{\text {in }}}\right|=\left|\frac{A_{\text {out }}}{A_{\text {in }}}\right|^{2}=\frac{L^{2}\left|A_{2}\right|^{2}+\left|A_{1}\right|^{2}+i L\left(A_{2}^{*} A_{1}-A_{1}^{*} A_{2}\right)}{L^{2}\left|A_{2}\right|^{2}+\left|A_{1}\right|^{2}-i L\left(A_{2}^{*} A_{1}-A_{1}^{*} A_{2}\right)} .
$$

For $\ell=0$ and $\omega \rightarrow 0$, the $\left|A_{2}\right|^{2}$ term in Eq. (21) is evaluated by 


$$
\begin{aligned}
\left|A_{2}\right|^{2} & =\left|C_{\mathrm{in}}\right|^{2} \frac{r_{s h}^{4-2 \beta} \Gamma(1-2 \alpha) \Gamma(1+2 \alpha) \Gamma^{2}(2 \beta-2)}{\Gamma\left(\omega_{a}-\alpha\right) \Gamma\left(1+\omega_{a}-\alpha\right) \Gamma\left(\omega_{a}+\alpha\right) \Gamma\left(1+\omega_{a}+\alpha\right)} \\
& \approx\left|C_{\mathrm{in}}\right|^{2} r_{s h}^{2},
\end{aligned}
$$

by using $\Gamma(z) \Gamma(1-z)=\pi / \sin \pi z$. This approximation means that the Compton wavelength of the scattered wave is much greater than the sonic black hole size. Furthermore, the dominant contribution of $s$-mode is the case of vanishing angular potential $(\ell=0)$ of the whole propagation modes [18]. Similarly, the $\left|A_{1}\right|^{2}$ term can be calculated as $\left|A_{1}\right|^{2} \approx\left|C_{\text {in }}\right|^{2} r_{s h}^{4}$ in the same limit used in Eq. (22). Provided that this is compared with Eq. (22), $\left|A_{1}\right|^{2}$ is negligible for the small sonic black hole horizon.

On the other hand, the non-trivial contribution of the reflection coefficient arises from the term of $A_{2}^{*} A_{1}-A_{1}^{*} A_{2}$. Using $\Gamma(z) \Gamma(1-z)=\pi / \sin \pi z$, we obtain

$$
A_{2}^{*} A_{1}-A_{1}^{*} A_{2} \approx i \frac{r_{s h}^{2} \omega\left|C_{\mathrm{in}}\right|^{2}}{2 a}
$$

From Eqs. (22) and (23), the reflection coefficient is simply expressed by

$$
\mathcal{R}^{\ell=0} \approx \frac{2 a L-\omega}{2 a L+\omega}
$$

which is expected to have a range with $0 \leq \mathcal{R} \leq 1$ by definition. This fact restricts the frequency of $\omega$ to $0 \leq \omega \leq 2 a L$. The absorption coefficient can be obtained by the reflection coefficient since we have $1-\mathcal{R}=\mathcal{A}$, which produces $\mathcal{A}^{\ell=0}=2 \omega /(2 a L+\omega)$. From the definition of absorption coefficient in Eq. (19), we also obtain $\mathcal{A}^{\ell=0}=\omega / 2 L^{2}(2 a L+\omega)$. Comparing these both relations determines the positive dimensionless constant, $L=1 / 2$. As a consequence, the reflection and the absorption coefficients are expanded as

$$
\mathcal{R}^{\ell=0}=1-\frac{2 \omega}{a}+\mathcal{O}\left(\omega^{2}\right), \quad \mathcal{A}^{\ell=0}=\frac{2 \omega}{a}+\mathcal{O}\left(\omega^{2}\right)
$$

and their behaviors are plotted in the FIG. 2. Note that the critical value of $a_{\mathrm{c}}=2 \omega$ exists, which gives "the total absorption" $\left(\mathcal{A}^{\ell=0}=1\right)$ and "the no reflection" $\left(\mathcal{R}^{\ell=0}=0\right)$. This signifies that we have to adjust the relation between the velocity and the energy of phonons, when we control the velocity of the fluid in the experimental system. 

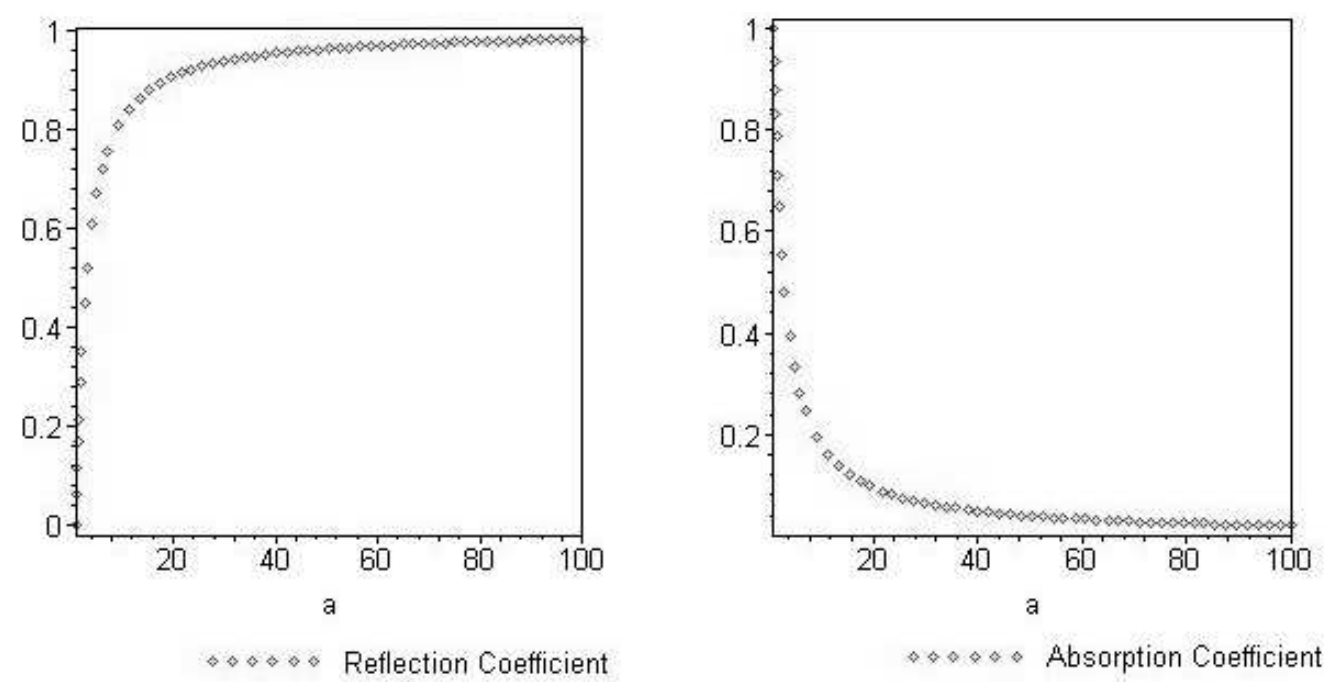

FIG. 2. Plots of the reflection and the absorption coefficients for $\omega=1$. The valid range of $a$ is $a \geq 1$ for the fixed $\omega=1$.

On the other hand, a relation between the vacuum expectation value of the number operator and the reflection coefficient can be given in the form $[15,16]$,

$$
<0|N| 0>=\left(\left|\frac{A_{\text {in }}}{A_{\text {out }}}\right|^{2}-1\right)^{-1}=\frac{\mathcal{R}}{1-\mathcal{R}} .
$$

Finally, the Hawking temperature is defined by

$$
<0|N| 0>=\frac{1}{\exp \left(\omega / T_{H}\right)-1}
$$

and combining this with Eq. (25) leads to the Hawking temperature as

$$
\left.T_{H}\right|_{\omega \rightarrow 0} \approx \frac{a}{2}
$$

This result coincides with the well-known result of the Hawking temperature in that it depends only upon the flow divergence $(a)$ passing through the sonic horizon surface at a certain time, which is closely related to surface gravity on the horizon. The absorption cross section for the dominant s-wave mode is given by $\sigma_{\mathrm{abs}}^{\ell=0}=\mathcal{A} / \omega=2 /(a+\omega)$ with the help of Eq. (25), and the decay rate of the sonic horizon can be easily calculated by

$$
\Gamma_{\text {decay }}^{\ell=0}=\left.\frac{\sigma_{\text {abs }}^{\ell=0}}{e^{\omega / T_{H}}-1}\right|_{\omega \rightarrow 0} \approx \frac{4}{\omega} .
$$


The system we are considering is only for the low-energy frequency of the phonon field. However, at high frequencies, there exist a kind of backscattering from the geometry of the acoustic black hole. More precisely, this is equivalent to the case of $\omega^{2} \geq 4 a^{2}$ in our approach, which yields an imaginary value of $\beta$. A straightforward calculation following a similar procedure at high frequencies produces the exact results for the reflection and the absorption coefficients,

$$
\begin{aligned}
\mathcal{R}_{\omega>>} & =\frac{\cosh \pi \omega_{a}^{-}+\cos j_{\ell} \pi}{\cosh \pi \omega_{a}^{+}+\cos j_{\ell} \pi} \\
\mathcal{A}_{\omega>>} & =\frac{\cosh \pi \omega_{a}^{+}-\cosh \pi \omega_{a}^{-}}{\cosh \pi \omega_{a}^{+}+\cos j_{\ell} \pi}
\end{aligned}
$$

respectively. Note that $\omega_{a}^{ \pm}=\omega / a \pm \sqrt{\omega^{2}-4 a^{2}} / a$ and $j_{\ell}=\sqrt{1-\frac{2 c}{a r_{s h}} \ell(\ell+1)}$ and it is easy to show that $\mathcal{R}_{\omega>>}+\mathcal{A}_{\omega>>}=1$. Since $\omega_{a}^{+} \approx 2 \omega / a$ and $\omega_{a}^{-} \approx 0$ at high frequency region, the reflection $(\mathcal{R})$ and the absorption coefficients $(\mathcal{A})$ are

$$
\begin{aligned}
& \mathcal{R}_{\omega>>}=\left.\frac{1+\cos j_{\ell} \pi}{\cosh 2 \pi \omega a+\cos j_{\ell} \pi}\right|_{\omega>>} \rightarrow 0, \\
& \mathcal{A}_{\omega>>}=\left.\frac{\cosh 2 \pi \omega a-1}{\cosh 2 \pi \omega a+\cos j_{\ell} \pi}\right|_{\omega>>} \rightarrow 1 .
\end{aligned}
$$

Note that this behavior is nothing but a scattering problem in the flat spacetime background. In the tortoise coordinate system, $r^{*}=\int d r / \sigma(r)$, one may read off the effective potential given by $V_{\text {eff }}(r)=\omega^{2}-V_{\ell}(r)$ from the Wheeler-Regge equation, where

$$
V_{\ell}(r)=\sigma(r)\left[\frac{\partial_{r} \sigma(r)}{r}+\frac{\ell(\ell+1)}{r^{2}}\right]
$$

and $\sigma(r)$ is a metric function in the metric, $(d s)^{2}=-\sigma(r) d t^{2}+\sigma^{-1}(r) d r^{2}+d^{2} \Omega$. It is easily shown that the effective potential of the wave equation becomes trivial as $V_{\text {eff }}(r)=\omega^{2}$ at high frequencies. Therefore, the Wheeler-Regge equation describes the free field equation with the constant effective potential of flat spacetime. Furthermore, the decay rate at high frequencies from Eq. (29) vanishes as $\omega$ goes to infinity, which is compatible with the statements at high frequencies as discussed before. 


\section{ACKNOWLEDGMENTS}

J.J.O. would like to thank Edwin J. Son, Jaehoon Kim, and Hyunjoo Lee for exciting and valuable discussions and he is grateful to Yoonbai Kim, Hyeonjoon Shin, and Gungwon Kang for a warm hospitality while he was staying at SKKU and KIAS as a visiting scholar. He also wishes to thank Russell J. Smith for helpful comments on this paper. This work was supported by the Basic Science Research Program of the Korean Science \& Engineering

Foundation Grant. At the final stage of this work, J.J.O. was supported by the Post-doctoral Fellowship Program of Korea Science \& Engineering Foundation (KOSEF). 


\section{REFERENCES}

[1] S. W. Hawking, Nature, 248 (1974) 30.

[2] W. G. Unruh, Phys. Rev. Lett. 46 (1981) 1351.

[3] M. Novello, M. Visser, and G. Volovik, Artificial black holes, World Scientific, River Edge, USA (2002).

[4] S. Corley and T. Jacobson, Phys. Rev. D57 (1998) 6269.

[5] T. Jacobson and G. E. Volovik, Phys. Rev. D58 (1998) 064021.

[6] T. Jacobson and D. Mattingly, Phys. Rev. D61 (1999) 024017.

[7] W. G. Unruh, Phys. Rev. D51 (1995) 2827.

[8] W. G. Unruh and R. Schützhold, Phys. Rev. D68 (2003) 024008.

[9] M. Visser, Class. Quantum Grav. 15 (1998) 1767.

[10] S. Liberati, S. Sonego, and M. Visser, Class. Quantum Grav. 17 (2000) 2903.

[11] C. Barceló, S. Liberati, and M. Visser, Class. Quantum Grav. 18 (2001) 1137.

[12] R. Balbinot, S. Fagnocchi, and A. Fabbri, Quantum Effects in Acoustic Black Holes:The Backreaction, [gr-qc/0405098]; R. Balbinot, S. Fagnocchi, A. Fabbri, and G. P. Procopio, Backreaction in Acoustic Black Holes, [gr-qc/0405096].

[13] M. Abramowitz and I. A. Stegun, Handbook of Mathematical Functions (Dover Publications Inc., New York, ninth printing, 1970).

[14] D. Birmingham, I. Sachs, and S. Sen, Phys. Lett. B413 (1997) 281.

[15] K. Ghoroku and A. L. Larsen, Phys. Lett. B328 (1994) 28.

[16] W. T. Kim and J. J. Oh, Phys. Lett. B461 (1999) 189.

[17] S. Das, G. Gibbons, and S. Mathur, Phys. Rev. Lett. 78 (1997) 417. 
[18] W. T. Kim, J. J. Oh, and K. H. Yee, Phys. Rev. D66 (2002) 044017. 